Document downloaded from:

http://hdl.handle.net/10251/120663

This paper must be cited as:

Gimenez Gandia, JJ.; Sung, KW.; Gomez-Barquero, D. (2018). Semianalytical Approach to the PDF of SINR in HPHT and LPLT Single-Frequency Networks. IEEE Transactions on Vehicular Technology. 67(5):4173-4181. https://doi.org/10.1109/TVT.2018.2791347

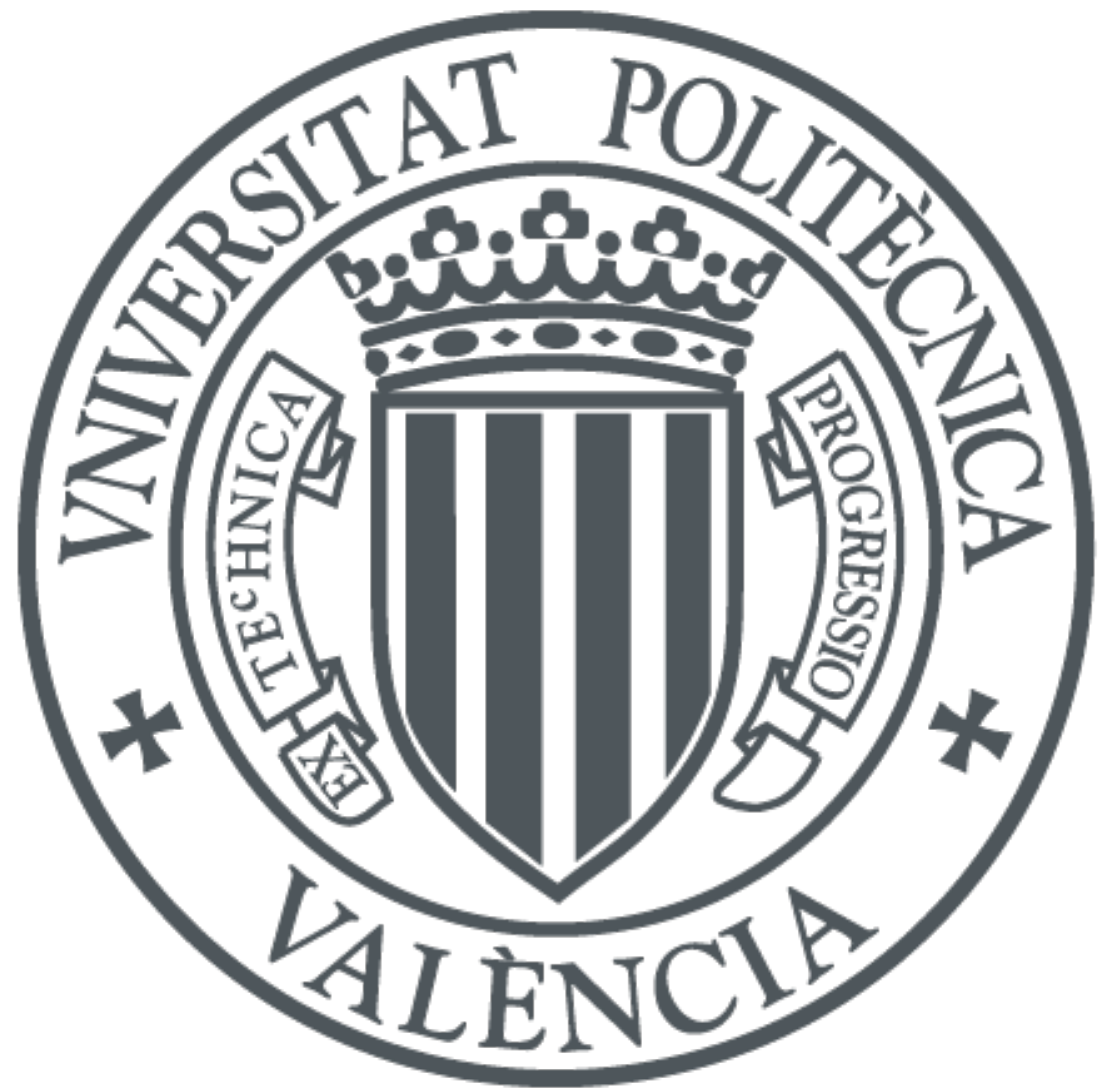

The final publication is available at

http://doi.org/ 10.1109/TVT.2018.2791347

Copyright Institute of Electrical and Electronics Engineers

Additional Information

(c) 2018 IEEE. Personal use of this material is permitted. Permission from IEEE must be obtained for all other users, including reprinting/ republishing this 


\title{
Semianalytical Approach to the PDF of SINR in HPHT and LPLT Single Frequency Networks
}

\author{
Jordi Joan Gimenez, Ki Won Sung, and David Gomez-Barquero
}

\begin{abstract}
Single Frequency Networks (SFN) are widely adopted in terrestrial broadcast networks based on High-Power High-Tower (HPHT) deployments. The mobile broadcasting standard Evolved Multimedia Broadcast Multicast Service (eMBMS) also enables SFN operation but with limited performance due to short cyclic prefix (CP) duration, suitable for dense Low-Power Low-Tower (LPLT) networks. An enhancement of the eMBMS specification, by introducing larger CP duration, may allow for the deployment of large area SFNs and even the combined operation of HPHT and LPLT stations. The knowledge of the SINR distribution over an SFN area may facilitate the selection of transmission parameters according to the network topology. This paper presents a semianalytical method for the calculation of the SINR distribution in SFNs with low computational complexity compared to Monte-Carlo simulations. The method, which builds on previous work developed for cellular communications, is applied to HPHT+LPLT SFNs and evaluated against different transmission and network parameters.
\end{abstract}

Index Terms-Single Frequency Network (SFN), network planning, low-power low-tower (LPLT), high-power high-tower (HPHT), cyclic prefix

\section{INTRODUCTION}

$\mathbf{S}$ INGLE Frequency Networks (SFN) enable simultaneous transmission across a set of stations using the same frequency. When deployed over HPHT (high-power hightower) networks, long cyclic prefix (CP) or guard interval (GI) duration is required to counteract inter-symbol interference (ISI) caused by artificial e choes from co-channel stations [1]. The terrestrial broadcasting standards DVB-T2 (Digital Video Broadcasting Terrestrial $2^{\text {nd }}$ Generation) [2] and ATSC 3.0 (Advanced Television Systems Committee) [3] enable maximum CP duration of $532 \mu$ s and $703 \mu$ s with 32k FFT $8 \mathrm{MHz}$ and $6 \mathrm{MHz}$, respectively. These standards offer an ISI-free maximum distance between stations of about $160 \mathrm{~km}$ and 210 $\mathrm{km}$.

eMBMS (Evolved Multimedia Broadcast Multicast Service) [4] is specified i n 3 GPP ( $3^{\text {rd }} \mathrm{G}$ eneration Partnership Project) Release 13 with a shorter CP $(33.3 \mu \mathrm{s}, 10 \mathrm{~km})$ to fit $t$ he $m$ uch $d$ ense $n$ ature of L PLT ( low-power low-tower) cellular networks. Note, however, that this specification lacks of signaling to identify which sub-frames use the $33.3 \mu \mathrm{s} \mathrm{CP}$

This work was partially supported by the Ministerio de Educación y Ciencia, Spain (TEC2014-56483-R), co-funded by European FEDER funds.

Jordi Joan Gimenez and David Gomez-Barquero are with the iTEAM Research Institute of the Universitat Politecnica de Valencia (iTEAM-UPV), Valencia, Spain. e-mail: \{jorgigan,dagobar\}@iteam.upv.es

Ki Won Sung is with the Communication Systems Department at the School of ICT at KTH Royal Institute of Technology, Kista, Sweden. email:sungkw@kth.se so it can not be configured. eMBMS has been enhanced in Release 14 [5] to support larger inter-site distances (ISDs) by the introduction of a $200 \mu \mathrm{s} \mathrm{CP}$ (up to $60 \mathrm{~km}$ ), which could allow for SFN operation with combined HPHT and LPLT infrastructure.

The interest in such mixed deployments has recently led to several studies. The authors in [6] investigate the performance of eMBMS in SFNs as a function of the cell radius and the CP length. Reference [7] presents a comparison between the coverage and cost for HPHT, LPLT, and mixed HPHT+LPLT deployments. Studies in [8] have studied the achievable spectral efficiency for DTT provision using cellular networks.

The SINR (Signal-to-Interference plus Noise Ratio) distribution and, in particular, its CDF (Cumulative Distribution Function) are interesting to characterize a service area. The shape of the CDF (and in particular the slope at tail percentages) provides knowledge of the required SINR to reach a certain coverage percentage. The implications of particular network characteristics (e.g. inter-site distance) or transmission parameters (e.g. CP duration) can be observed in the SINR distribution over an SFN as well as the effects of the so-called SFN gain.

The calculation of the SINR distribution over large SFN areas involves a high time-consuming process based on extensive Monte-Carlo (MC) simulations to achieve accurate results. This motivates us to investigate a low-complexity analytic method. As for heterogeneous cellular networks which resemble the mixed HPHT and LPLT deployment, analytic methods for SINR distribution have been developed by employing stochastic geometry [9]-[11]. However, these works assume random positions of base stations under Rayleigh fading environment. Thus, the results cannot be applied to the network planning of large-scale SFN where the locations of transmit towers are carefully chosen and the radio propagation is heavily influenced by shadow fading.

The main contribution of this paper is to develop and apply a semianalytical method for estimating the SINR distribution in SFN areas using mixed HPHT+LPLT stations. The method builds on [12] where the downlink SINR distribution of homogeneous femto-cell deployment is approximated. With the method, we evaluated the SINR distribution of mixed HPHT+LPLT deployments against key planning parameters such as the CP duration and ISD. The methodology may serve as a coarse approximation to the selection of proper parameters in real networks as well as a way of understanding their effects over SFN areas.

The paper is organized around the following sections: Section II presents the derivation of the PDF of the SINR 
for SFN networks. Section III presents the methodology and simulation setup for HPTH and LPLT deployments. Section IV covers the validation of the methods by comparison to MC simulations. Section V analyses the effect of the SFN configuration in HPHT and LPLT networks under a realistic scenario. Conclusions are presented in Section VI.

\section{Derivation of the PDF of THE SINR IN SFN NETWORKS}

The process for deriving the PDF of the SINR in a service area has been divided into four steps. The general expressions of received power and thermal noise are obtained first. Second, the SINR for Multiple Frequency Network (MFN) and SFN are calculated as a function of the receiver position. Third, the statistics of the SINR are determined and, finally, the expression of the PDF of the SINR in a given service area is derived.

\section{A. Received Signal and Noise}

The received power $P_{r}(\mathrm{~W})$ from a given transmitter can be expressed as a log-normally distributed random variable (RV) in terms of the available field strength $E_{r}(\mu \mathrm{V} / \mathrm{m})$ at the receiver location as:

$$
P_{r}=\exp \left(\ln \left(\frac{E_{r}^{2}}{480 \pi^{2}} \frac{c^{2}}{f^{2}} \frac{G_{r}}{L_{T}}\right)+\beta X_{l}\right)
$$

where $f$ is the frequency in $\mathrm{Hz}, c$ is the speed of light in $\mathrm{m} / \mathrm{s}$, $G_{r}$ is the receiving antenna gain and $L_{T}$ includes other loss factors such as feeder loss, height loss (for portable reception) or building penetration loss (for indoor reception), where applicable. $\beta=\ln (10) / 10$, where $\ln ()$ denotes the natural logarithm. Note that $E_{r}$ depends on the ERP (Equivalent Radiated Power) and path loss, which is described by a proper propagation model. $X_{l}$ is a RV that models the locationdependent shadow fading which is generally assumed to be log-normal distributed (Gaussian in $\mathrm{dB}$ ) with zero mean and standard deviation $\sigma_{X_{l}}$ [13].

The PDFs of $P_{r}$ is described by:

$$
f_{P_{r}}(z)=\frac{1}{z \sigma_{P_{r}} \sqrt{2 \pi}} \exp \left(\frac{-\left(\ln z-\mu_{P_{r}}\right)^{2}}{2 \sigma_{P_{r}}^{2}}\right)
$$

where $\mu_{P_{r}}=\ln \left(\frac{E_{r}^{2}}{480 \pi^{2}} \frac{c^{2}}{f^{2}} \frac{G_{r}}{L_{T}}\right)$ and $\sigma_{P_{r}}^{2}=\beta^{2} \sigma_{X_{P_{r}}}^{2}$.

Noise power, $N$, can be considered as a constant value which power depends on the Boltzmann constant $k$, the ambient temperature in Kelvin $T$, the system bandwidth $W$ and the noise figure of the receiver $F$. An auxiliary Gaussian $\mathrm{RV} X_{n}$ with zero mean and zero variance is introduced so that $N$ can be treated as well as a log-normal RV as:

$$
N=\exp \left(\ln (k T B F)+X_{n}\right)
$$

\section{B. SINR in Multiple and Single Frequency Networks}

In an MFN, signals are broadcast from station in an asynchronous manner so that neighboring transmitters must use a different frequency in order to keep interference low. At a certain location, signal from the desired transmitter as well as signals from the co-channel transmitters, which are treated as interference, are received. The SINR experienced at a given receiver location can be expressed as:

$$
\gamma_{M F N}=\frac{P_{r_{0}} Q_{\varphi_{0}}}{\sum_{j=1}^{M-1} P_{r_{j}} Q_{\varphi_{j}}+N}
$$

where $P_{r_{j}}$ denotes the received power from transmitter $j$, $Q_{\varphi_{j}}$ is the receiver antenna pattern attenuation relative to the pointing direction between transmitter and receiver. $M$ is the total number of transmitters in the MFN where $M$ 1 create interference. The denominator yields to the sum of the interference powers and background noise power, which corresponds to the sum of $M \log$-normal RVs.

In SFNs, a cluster of transmitters use the same frequency to transmit the same signal in a synchronous manner. Thanks to the properties of the OFDM, receivers can constructively combine part of the signals coming from different transmitters, at different distances and delays. This behaviour is modeled by equation (5), according to [14].

$$
w\left(\Delta_{t}\right)=\left\{\begin{array}{cl}
1 & 0<\Delta_{t} \leq T_{g} \\
\left(\frac{\left(T_{u}+T_{g}\right)-\Delta_{t}}{T_{u}}\right)^{2} & T_{g}<\Delta_{t}<T_{p} \\
0 & \Delta_{t}>T_{p}
\end{array}\right.
$$

As the delay between contributions $\left(\Delta_{t}\right)$ increases beyond CP duration $\left(T_{g}\right)$ SFN self-interference appears. The constructive contribution decreases with a quadratic law until a certain delay is reached $\left(T_{p}\right)$. Beyond this delay the contribution is fully destructive. $T_{p}$ is the equalization interval. In the case of pilot-aided channel estimation, this corresponds to the interval in which channel estimation is correctly performed. In practice, receivers can resolve delays up to the equalization interval, which is a fraction of the Nyquist limit $T_{N y}$ (i.e. 57/64 according to [14]). $T_{N y}$ is defined as the maximum delay spread between two contributions so that channel estimation can be performed. When combined time and frequency interpolation (2D interpolation) can be performed, the Nyquist limit is given as $T_{N y}=T_{u} / D_{x}$, where $T_{u}=1 / \Delta f$ is the useful symbol duration (which depends on the sub-carrier spacing $\Delta f$ ) and $D_{x}$ is the spacing between sub-carriers carrying a pilot in the frequency direction.

According to $w\left(\Delta_{t}\right)$, the SINR at a given receiver location in an SFN can be expressed as:

$$
\gamma_{S F N}=\frac{\sum_{j=1}^{M} w_{j}\left(\Delta_{t}\right) P_{r_{j}} Q_{\varphi_{j}}}{\sum_{j=1}^{M}\left[1-w_{j}\left(\Delta_{t}\right)\right] P_{r_{j}} Q_{\varphi_{j}}+N}
$$

As for the MFN, numerator and denominator in (6) also define the summation of log-normal RVs. However, for the SFN, part of power $\left(P_{r_{j}}\right)$ is present at both parts of the fraction, which are not fully independent. The influence of the correlation in the SINR distribution was studied in [1] and is discussed in Section IV-A. 


\section{Approximating the sum of log-normal distributions}

The calculation of the PDF of the SINR in MFNs and SFNs involves the summation of log-normal RVs whose exact closed-form expression is still unknown. Most approximations in the literature assume that the distribution of the summation of log-normal distributions is also log-normal [15]. The Fenton-Wilkinson (FW) method [16] provides a closedform for the calculation that matches the first two moments of the power sum of log-normal distributions to the first two moments of another log-normal distribution $\left(\mu_{V}\right.$ and $\sigma_{V}^{2}$ ). The Schwartz and Yeh method [17] uses a polynomial approximation to determine the parameters of the resultant distribution. Safak [18] and Beaulieu-Xie [19] use analytical expressions. Other more elaborated methods such as [20] specify a compound distribution.

In this paper, the FW is chosen due to its good balance between computation complexity and accuracy. However, the methodology applies to other methods. FW is found to be accurate in the tail portion of the PDF which is useful for coverage estimation. Furthermore, FW has been adapted to terrestrial broadcasting SFNs, known as the k-LNM (lognormal method). k-LNM is an extension of the FW method that introduces a correction factor $k$ applied to the variance to improve the accuracy specially for high probability percentages [15].

The mean $\left(\mu_{\Psi}\right)$ and variance $\left(\sigma_{\Psi}^{2}\right)$ of the resultant lognormal distribution with the k-LNM method are given by (7) and (8) in which $k=1$ leads to the FW method. Notice the mutual dependence between the mean value and variance.

$$
\begin{gathered}
\sigma_{\Psi}^{2}=\ln \left(k \frac{\sum_{j=1}^{N} \exp \left(2 \mu_{j}+\sigma_{j}^{2}\right)\left(\exp \left(\sigma_{j}^{2}\right)-1\right)}{\left(\sum_{j=1}^{N} \exp \left(\mu_{j}+\frac{\sigma_{j}^{2}}{2}\right)\right)^{2}}+1\right) \\
\mu_{\Psi}=\ln \left(\sum_{j=1}^{N} \exp \left(\mu_{j}+\frac{\sigma_{j}^{2}}{2}\right)\right)-\frac{\sigma_{\Psi}^{2}}{2}
\end{gathered}
$$

\section{PDF of SINR in MFNs and SFNs}

Assuming that (4) and (6) define a ratio between independent log-normal distributions, the SINR $(\gamma)$ can be regarded as a $\log$-normal distribution. If the receiver location $\rho$ (defined in polar coordinates $r_{y}, \theta_{y}$ ) is uniformly distributed over the circular service area of radius $R$, the joint PDF of the SINR and the receiver location can be expressed as:

$$
f_{\gamma, \rho}\left(z, r_{y}, \theta_{y}\right)=\frac{1}{z \sigma_{\gamma_{y}} \sqrt{2 \pi}} \exp \left(\frac{-\left(\ln z-\mu_{\gamma_{y}}\right)^{2}}{2 \sigma_{\gamma_{y}}^{2}}\right) \frac{r_{y}}{\pi R^{2}}
$$

The marginal PDF can be calculated by the integration of (9) over the coordinate axis $r_{y}$ and $\theta_{y}$, in the intervals $0 \leq r_{y} \leq R$ and $0 \leq \theta_{y} \leq 2 \pi$, as:

$f_{\gamma}(z)=\int_{0}^{R} \int_{0}^{2 \pi} \frac{r_{y}}{z \sigma_{\gamma_{y}} R^{2} \sqrt{2 \pi^{3}}} \exp \left(\frac{-\left(\ln z-\mu_{\gamma_{y}}\right)^{2}}{2 \sigma_{\gamma_{y}}^{2}}\right) \mathrm{d} \theta_{y} \mathrm{~d} r_{y}$
Equation (10) presents the generic form of the marginal PDF. Note that $\mu_{\gamma_{y}}$ and $\sigma_{\gamma_{y}}^{2}$ are function of the receiver location $\left(r_{y}, \theta_{y}\right)$ and can be particularized for $\gamma_{M F N}$ or $\gamma_{S F N}$ as follows.

For MFNs, $\mu_{\gamma_{y}}=\mu_{P_{r}}-\mu_{\Psi_{\Sigma_{M}}}$ and $\sigma_{\gamma_{y}}^{2}=\sigma_{P_{r}}^{2}+\sigma_{\Psi_{\Sigma_{M}}}^{2}$. In this case $\mu_{\Psi_{\Sigma_{M}}}$ and $\sigma_{\Psi_{\Sigma_{M}}}^{2}$ refer to the summation of $M-1$ interfering signals plus noise, which result in a clear dependence on the receiver position since each interfering contribution is characterized by location-dependent path loss and receiver antenna pattern attenuation.

For SFNs, $\mu_{\gamma_{y}}=\mu_{\Psi_{\Sigma_{w}}}-\mu_{\Psi_{\Sigma_{1-w}}}$ and $\sigma_{\gamma_{y}}^{2}=\sigma_{\Psi_{\Sigma_{w}}}^{2}+$ $\sigma_{\Psi_{\Sigma_{1-w}}}^{2}$, where $\Sigma_{w}$ refers to the summation of log-normal RVs of the constructive contributions in the SFN whereas $\Sigma_{1-w}$ to the summation of the interfering contributions and noise; corresponding to the numerator and denominator of (6). Again, each signal contributes with a field strength that depends on path loss and the angular discrimination of the antenna pattern according to receiver position.

The PDF can be obtained by means of numerical integration methods.

\section{Methodology and Simulation Setup}

The PDF given in (10) is numerically calculated under certain network characteristics and compared with the result provided by an MC simulation. The result will provide information about the accuracy of the methodology introduced in Section II.

The networks to be studied are modeled by means of a hexagonal-cell lattice. HPHT and LPLT cells define circles of radius $R_{H P}$ and $R_{L P}$, respectively. Assumed inter-site distances are $I S D_{H P}=60 \mathrm{~km}$ and $I S D_{L P}=5,10$ and $20 \mathrm{~km}$ with an station located at the center of each cell. The cell of interest $(\mathrm{CoI})$ is the central cell of the network around which the neighboring cells are deployed by adding tiers.

Three different types of network deployments are considered (see Figure 1): pure HPHT, pure LPLT and mixed HPHT+LPLT. HPHT networks are deployed under MFN and SFN configurations whereas LPLT networks are only considered under SFN conditions:

- The HPHT MFN is a reference case for current DTT deployments not using SFNs. A certain frequency reuse $(N=1,3$, and 4$)$ pattern is chosen for comparison. According to $N$, the co-channel cells are located at a certain reuse distance to the CoI.

- The HPHT SFN is a large area SFN in which all transmitters in the network transmit the same signal using the same frequency. The CoI is at the center of the SFN. The $\mathrm{CP}$ duration defines the distance beyond which signals are destructive.

- The LPLT SFN consist of a cluster of LPLT stations within the area of a HPHT cell (CoI) that constitute an SFN. The same frequency reuse of the HPHT MFN case is applied for comparison.

- The HPHT+LPLT SFN consists of the previous network, but the HPHT station in the center of the CoI is also active. Both LPLTs and the HPHT create an SFN. 

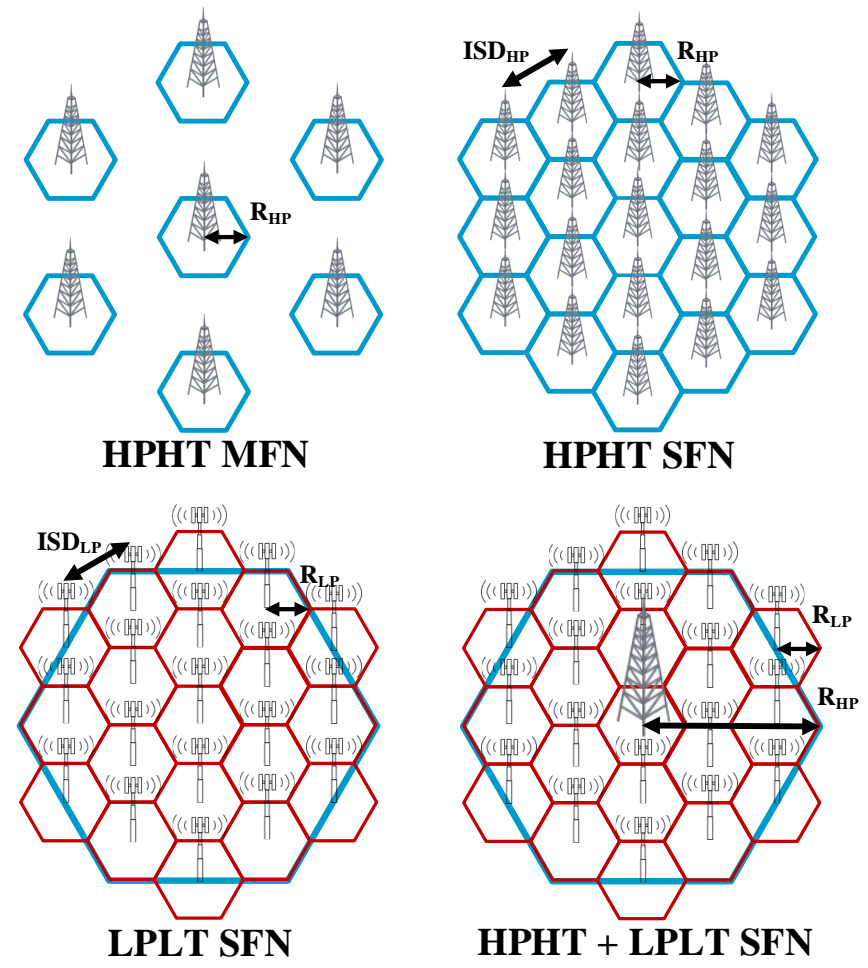

Fig. 1. Ideal hexagonal network deployments constituting a HPHT MFN with frequency reuse $N=4$ (top left), a HPHT SFN (top right), a LPLT SFN covering one HPHT cell (bottom left) and mixed HPHT+LPLT covering one HPHT cell (bottom right).

The characteristics of the network elements as well as relevant considerations for the simulation setup are described in Table I. These parameters are in line with those recommended by EBU [21] for the simulation of theoretical HPHT and LPLT networks.

The antenna pattern of all transmitter stations is omnidirectional and the antenna height is set to $250 \mathrm{~m}$ for HPHT and $30 \mathrm{~m}$ for LPLT.

Regarding propagation, the ITU-R P.1546 [22] is generally used in terrestrial broadcasting to calculate the available field strength at the receiver. The calculation method is based on interpolation/extrapolation from field-strength curves which are empirically derived from measurements. The curves are given as function of distance, frequency $(650 \mathrm{MHz})$, effective antenna height, and time availability (P.1546 recommends taking the field strength exceeded in 50\% time for wanted and $1 \%$ for unwanted signals).

Roof-top fixed reception (10 m high) and its corresponding receiver antenna characteristics are considered $\left(11 \mathrm{~dB}_{D}\right.$ antenna gain and discrimination according to ITU-R BT.419 [23]). Receiver antenna points toward the center of the strongest cell in the network. The synchronization strategy for the FFT windows is "strongest signal" as described in [24]. For the SFN cases, CP duration of $33 \mu \mathrm{s}, 100 \mu \mathrm{s}, 200 \mu \mathrm{s}$ and $400 \mu$ s are studied.
TABLE I

NETWORK CHARACTERISTICS AND TRANSMITTER \& RECEIVER PARAMETERS

\begin{tabular}{|c|c|}
\hline Parameter & HPHT \\
\hline Frequency & $650 \mathrm{MHz}$ \\
\hline Reception Type & Fixed rooftop at $10 \mathrm{~m}$ \\
\hline Propagation Model & ITU-R P.1546-5 (Land) \\
\hline Signal time probability & $50 \%$ Wanted / $1 \%$ Interfering \\
\hline Shadow fading std dev. & $5.5 \mathrm{~dB}(100 \mathrm{~m} \times 100 \mathrm{~m})$ \\
\hline Transmit power & $10 \mathrm{~kW}$ ERP $\quad 40 \mathrm{~W}$ \\
\hline Transmit antenna pattern & Omni-directional \\
\hline Transmit antenna height & $250 \mathrm{~m} \quad 30 \mathrm{~m}$ \\
\hline Inter-site distance (ISD) & 5 to $20 \mathrm{~km}$ \\
\hline SFN synchronization strategy & Strongest signal \\
\hline Rx antenna model & ITU-R BT.419-3 \\
\hline $\mathrm{Rx}$ antenna gain & $11 \mathrm{~dB}_{D}$ \\
\hline Feeder loss & $4 \mathrm{~dB}$ \\
\hline Equivalent noise bandwidth & $10 \mathrm{MHz}$ \\
\hline Receiver noise figure & $6 \mathrm{~dB}$ \\
\hline CP duration & $33.3,100,200$ and $400 \mu \mathrm{s}$ \\
\hline Equalization Interval & $57 / 64$ of $T_{N y}$ \\
\hline Separation pilot-bearing carriers $\left(D_{x}\right)$ & 2 \\
\hline
\end{tabular}

\section{VALIDATION IN HPHT AND LPLT NETWORKS}

This section discusses the application of the proposed methodology to SFNs and HPHT and LPLT deployments. First, the probability distribution and the potential crosscorrelation of the random variables in the SINR numerator and denominator in SFNs are discussed. Next, the accuracy of two well-known methods for the summation of log-normal RVs will be assessed for HPHT and LPLT network deployments by comparison of the numerical calculations to $\mathrm{MC}$ simulations.

\section{A. Log-normal components in an SFN}

The equations of the SINR distribution derived in Section III assume that all involved RVs are log-normal distributed. In general, received power varies faster with location-dependent shadow fading than with distance. Coverage probability in terrestrial broadcasting is estimated assuming that received power is modelled as a log-normal random distribution within area elements ranging hundreds of meters (e.g $100 \mathrm{~m} \mathrm{x} 100$ m) [13]. However, in SFNs, the variation of the terms $w\left(\Delta_{t}\right)$ and $\left[1-w\left(\Delta_{t}\right)\right]$ needs to be investigated as well as the potential cross-correlation between SINR numerator and denominator elements in the delay interval in which part of a contribution is constructive and destructive [1].

An MC simulation is conducted in order to calculate the standard deviation of $w\left(\Delta_{t}\right)$ and $\left[1-w\left(\Delta_{t}\right)\right]$ within an area element of $100 \mathrm{~m}$ x $100 \mathrm{~m}$ which center is moved along the linear distance between two transmitters. With this, it is possible to know how $w\left(\Delta_{t}\right)$ affects the statistics of the SINR distribution in the critical region when part of the contribution is constructive and destructive. The variation of $w\left(\Delta_{t}\right)$ within an area element mainly depends on the selected transmitter mode, which defines $T_{g}, T_{u}$ and $D_{x}$. Table II shows a selection of sample parameters achieving CP duration of 33, 100, 200 
TABLE II

TRANSMISSION PARAMETERS FOR THE W $\left(\Delta_{t}\right)$ DISTRIBUTION ANALYSIS

\begin{tabular}{cccccc}
\hline \hline$T_{g}(\mu \mathrm{s})$ & $T_{u}(\mu \mathrm{s})$ & $T_{N y}(\mu \mathrm{s})$ & $T_{p}(\mu \mathrm{s})$ & $d_{g}(\mathrm{~km})$ & $d_{p}(\mathrm{~km})$ \\
\hline 33 & 133.33 & 66.66 & 60 & 9.9 & 18 \\
\hline 100 & 400 & 200 & 180 & 30 & 60 \\
\hline 200 & 800 & 400 & 360 & 60 & 120 \\
\hline 400 & 1600 & 800 & 720 & 120 & 240 \\
\hline
\end{tabular}

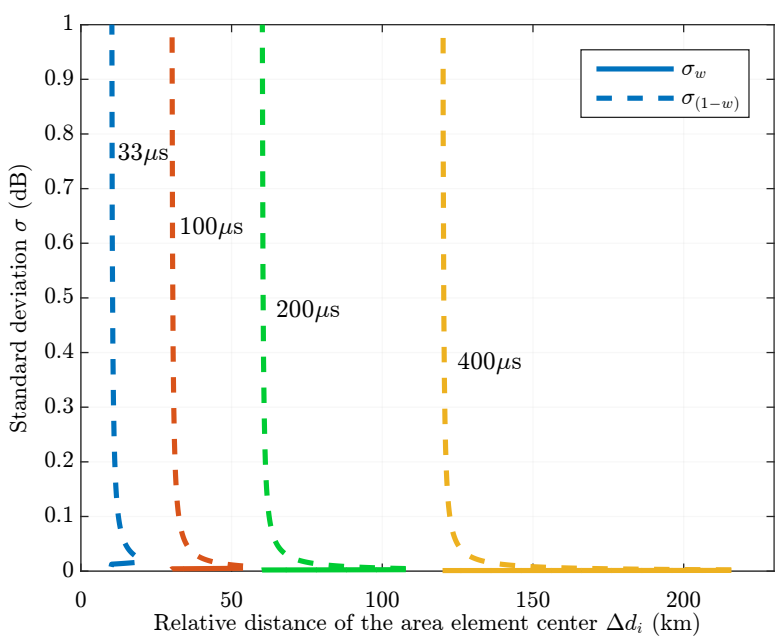

Fig. 2. Standard deviation of $w\left(\Delta_{t}\right)$ and $\left[1-w\left(\Delta_{t}\right)\right]$ for different $\mathrm{CP}$ duration $\left(T_{g}=33 \mu \mathrm{s}, 100 \mu \mathrm{s}, 200 \mu \mathrm{s}\right.$ and $\left.400 \mu \mathrm{s}\right)$ within a region of 100 $\mathrm{m} \times 100 \mathrm{~m}$ between two stations. Network parameters $T_{u}, T_{N y}$ and $T_{p}$ are selected according to Table II.

and $400 \mu$ s. The useful symbol duration as well as the Nyquist limit are accordingly extended. The relative distance between the transmitters can be directly related to the relative delay between the incoming signals by the speed of light $c$.

The results of the analysis, see Figure 2, show that at the left edge where the relative delay approaches $T_{g}$ the standard deviation is high whereas for other delays the standard deviation is below $0.1 \mathrm{~dB}$, which is lower than the typical $5.5 \mathrm{~dB}$ standard deviation of shadow fading. For the four $\mathrm{CP}$ duration values studied, the distance in which standard deviation is above 0.1 is around $2 \mathrm{~km}$ beyond $d_{g}$. Thus, the portion in which standard deviation remains low increases with CP duration. For larger area elements (e.g. $500 \mathrm{~m} \times 500 \mathrm{~m}$ ), the variability is higher in the critical region and the low standard deviation condition is not accomplished for a larger distance. For the upper bound, $T_{p}$, the standard deviation of the weighting function remains low what means that even with less dense pilot patterns the results will not be affected. The calculation of the correlation coefficient between the wanted and interfering components of the same signal show that both are fully correlated over the area element.

Assuming that the signal components are independent and that the only correlation exists due to the SFN selfinterference, the conventional estimate gives good accuracy. In the rest of the paper, it is assumed that $w\left(\Delta_{t}\right)$ and $\left[1-w\left(\Delta_{t}\right)\right]$ are constant within the area element so that all components in (6) can be treated as log-normally distributed.
TABLE III

Kullback-Leibler Distance Between the Monte-Carlo SIMULATION AND THE APPROXIMATION METHODS $\left(\mathrm{X}_{10}^{-3}\right)$

\begin{tabular}{lcccc}
\hline \hline & k-LNM5 & k-LNM7 & k-LNM9 & FW \\
\hline HPHT Network Scenarios & & & \\
\hline MFN $N=1$ & 0.9 & $\mathbf{0 . 3}$ & 1.4 & 2.5 \\
\hline MFN $N=3$ & 1.2 & $\mathbf{0 . 3}$ & 0.3 & 0.7 \\
\hline MFN $N=4$ & 1.3 & $\mathbf{0 . 3}$ & 0.4 & 0.5 \\
\hline SFN $T_{g}=33 \mu s$ & 17.7 & $\mathbf{2 . 2}$ & 13.0 & 24.7 \\
\hline SFN $T_{g}=100 \mu s$ & 15.7 & $\mathbf{1 . 7}$ & 14.5 & 27.6 \\
\hline SFN $T_{g}=200 \mu s$ & 14.5 & $\mathbf{2 . 4}$ & 18.7 & 33.9 \\
\hline SFN $T_{g}=400 \mu s$ & 12.4 & $\mathbf{4 . 6}$ & 27.3 & 48.5 \\
\hline LPLT Network Scenarios & & & \\
\hline SFN $T_{g}=33 \mu s$ & 20.8 & $\mathbf{1 2 . 3}$ & 48.2 & 78.5 \\
\hline SFN $T_{g}=100 \mu s$ & $\mathbf{1 4 . 7}$ & 24.5 & 98.5 & 166.8 \\
\hline SFN $T_{g}=200 \mu s$ & $\mathbf{2 2 . 8}$ & 27.1 & 119.9 & 190.9 \\
\hline SFN $T_{g}=400 \mu s$ & $\mathbf{1 2 . 0}$ & 27.9 & 113.9 & 191.8 \\
\hline
\end{tabular}

\section{B. Semianalytical Distributions with HPHT and LPLT}

The cummulative distribution functions (CDFs) of the SINR at the receivers within the CoI in HPHT and LPLT networks is computed using the k-LNM method with $k=0.5$ (k-LNM5), 0.7 (k-LNM7) and 0.9 (k-LNM9), as well as $k=1$ (FW). Corresponding $\mathrm{MC}$ simulations are computed for comparison. The regions of the curves linked to the coverage definition of the 95\% SINR availability are zoomed. The comparison between the PDFs obtained by the different methods is performed by calculating the Kullback-Leibler Distance (KLD), which measures the divergence between PDFs, as defined in [25]. The complete set of results are collected in Table III.

1) High-Power High-Tower MFNs and SFNs: Figure 3 depicts the CDFs of the SINR in the CoI for an MFN HPHT network with frequency reuse $(N=4)$. It can be seen that, the different methods provide similar results regarding the shape of the CDFs with differences below $0.1 \mathrm{~dB}$ among them. All methods deviate from the MC simulation in around $0.2 \mathrm{~dB}$. According to the KLD, k-LNM7 method is the closest to the MC simulation (see Table III). For the low probability region, the best approach is given by the FW method, although the differences among them are not critical.

Results have been also obtained for other frequency reuses $(N=1,3$, and 4$)$ showing a similar trend and accuracy for the different methods, as can be seen in Table III.

The same procedure is followed for HPHT SFNs with different $\mathrm{CP}$ duration. An example is shown in Figure 4 for a selected CP duration $T_{g}=100 \mu \mathrm{s}$. For the region above $50 \%$ probability the CDFs computed by different methods present a similar shape but large differences appear in the low probability region with differences up to $4 \mathrm{~dB}$ for the the 5\% value. The method that seems to best describe the probability distribution for such region is the KLNM-7, as it was previously obtained in the MFN case. Increasing $k$ yields to a pessimistic SINR distribution whereas the contrary occurs when decreasing this parameter. A similar trend is found for other CP duration although the divergence distance increases for duration below and above $100 \mu$ s (see Table III). In the 


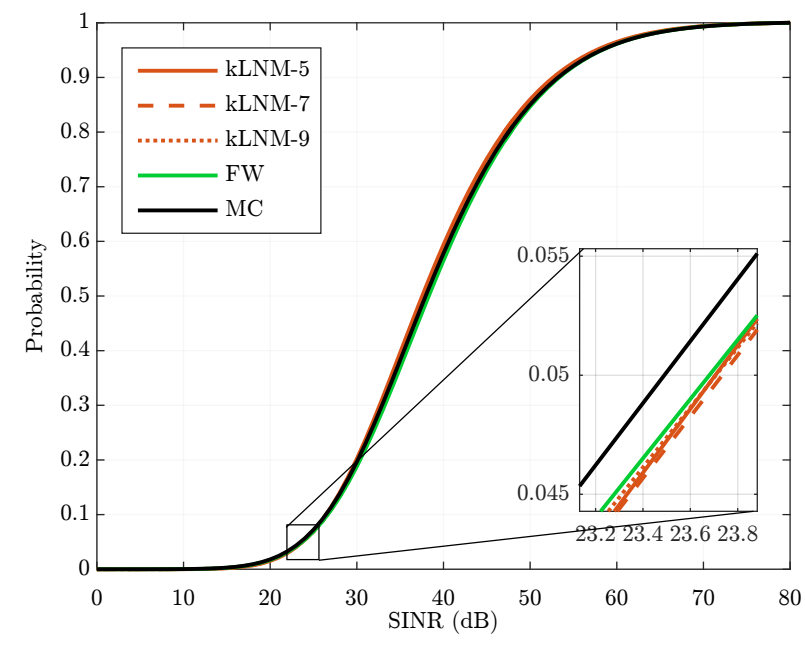

Fig. 3. CDFs for a HPHT MFN with frequency reuse $N=4$ approximated by means of the $\mathrm{k}$-LNM $(k=0.5,0.7$, and 0.9$)$ and the FW summation methods. The result of an MC simulation is also depicted.

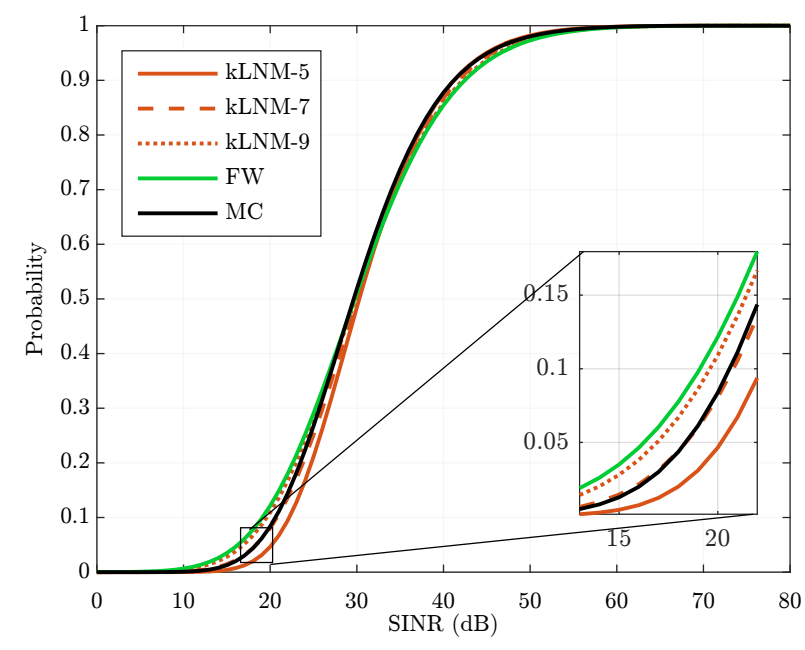

Fig. 4. CDFs for a large (self-interference limited) HPHT SFN approximated by means of the $\mathrm{k}-\mathrm{LNM}(k=0.5,0.7$, and 0.9$)$ and the FW summation methods. The result of a Monte-Carlo simulation is also depicted.

low probability region, the kLNM-7 method provides the most accurate result compared to the MC simulation.

According to the results obtained, the k-LNM method with $k=7$ is the most accurate for the calculation of MFNs and SFNs in HPHT networks. Compared to the MFN, the SFN calculation is less accurate than the application of the summation methods in the MFN cases.

2) Low-Power Low-Tower SFNs: For LPLT SFN deployments, Figure 5 shows the comparison between methods for a $T_{g}=33 \mu \mathrm{s}$ and an $I S D_{L P}$ of $5 \mathrm{~km}$. The CDFs present large differences in the low probability region. For this region, the kLNM7 approach is close up to $1 \mathrm{~dB}$ to the MC simulation. The k-LNM5 is the closest for even higher coverage percentages, although optimistic. According to the results in Table III for larger CP duration and the same $I S D_{L P}$, the best approach is provided by the k-LNM5 method, although the differences to $\mathrm{k}-\mathrm{LNM} 7$ are not large, being the later pessimistic for the

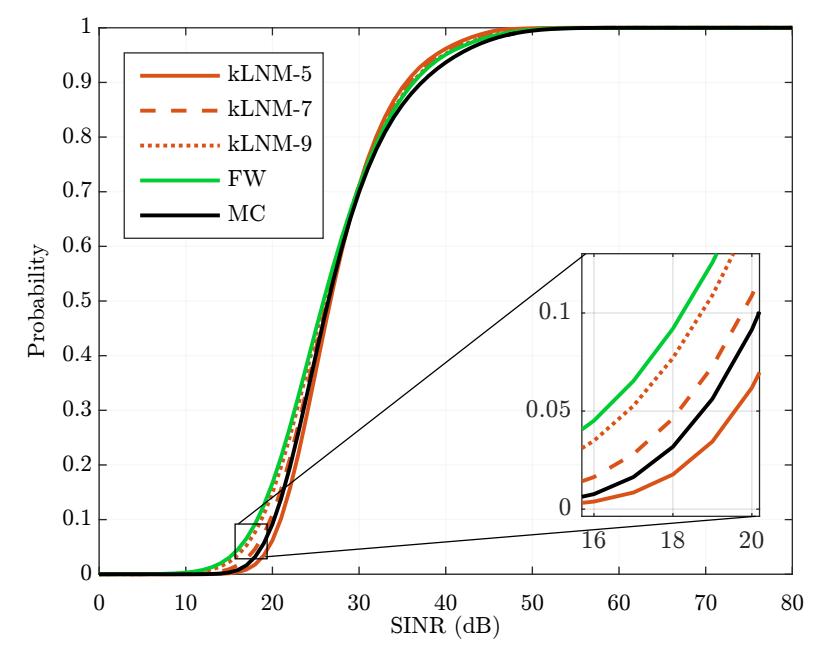

Fig. 5. CDFs for a LPLP SFN covering one HPHT cell approximated by means of different log-normal summation methods and as a result of a MonteCarlo simulation.

low probability region.

Compared to the methods for SFN HPHT deployments, it can be seen that, in general, all of them are less accurate than for the HPHT networks. However, they can provide an acceptable approach for a fast evaluation of the SINR over HPHT and LPLT cells.

3) Effects of the sum of distributions: The aggregation of signal contributions and its effect on the mean and standard deviation are the key points to understand the shape of the CDF curves. The aggregation of multiple log-normal contributions results in a log-normal distribution with decreased standard deviation and increased mean value.

In an MFN, the aggregation in the denominator of (4), results in a distribution with lower standard deviation than that of the individual contributions. The resultant SINR distribution is characterized by a larger standard deviation than that of the wanted signal. Thus, the CDF of the MFN is expected to present a low slope. In the case of an SFN, depending on the $\mathrm{CP}$ duration, the slope is different since adding more contributions in the numerator (for large CP duration) or denominator (short $\mathrm{CP}$ duration) of (6) will result in a lower standard deviation of the SINR. The overall effect results in the so-called SFN gain. An important factor on the value of the resultant standard deviation is the mean value of the individual contributions. The aggregation of signals with similar mean value provide a lower standard deviation than the aggregation of signals presenting more imbalances. On the other hand, the mean value of the resultant aggregation will be conditioned by the highest mean value within the contributions.

\section{SFN Network PlanNing in HPHT and LPLT NETWORKS}

This section delves into the characterization of the SINR distribution as a function of the network topology and the configuration of the transmission system. The study is centered on evaluating the shape of the PDF (and CDF) of the SINR distribution in traditional HPHT service areas and deployments 
TABLE IV

SINR (DB) IN HPHT MFNS AND SFNS AT DIFFERENT PERCENTILES

\begin{tabular}{|c|c|c|c|}
\hline & $10^{t h}$ & $5^{t h}$ & $1^{s t}$ \\
\hline \multicolumn{4}{|c|}{ Multiple Frequency Network } \\
\hline MFN $N=1$ & 5.6 & 2.2 & -3.8 \\
\hline MFN $N=3$ & 22.4 & 19.5 & 14.3 \\
\hline MFN $N=4$ & 26.6 & 23.7 & 18.5 \\
\hline \multicolumn{4}{|c|}{ Single Frequency Network } \\
\hline SFN $T_{g}=33 \mu s$ & 14.33 & 11.9 & 7.5 \\
\hline SFN $T_{g}=100 \mu s$ & 20.8 & 18.4 & 14.1 \\
\hline SFN $T_{g}=200 \mu s$ & 28.3 & 25.6 & 20.8 \\
\hline $\mathrm{SFN} T_{g}=400 \mu \mathrm{s}$ & 39.4 & 36.7 & 31.8 \\
\hline
\end{tabular}

using LPLT infrastructure exploiting the SFN concept. The critical parameters that are involved in the characteristics of the SFN involve the $I S D_{L P}$ and the CP duration. In addition, scenarios in which both kinds of infrastructures are combined are also studied.

\section{A. Comparison between HPHT MFNs vs HPHT SFNs}

This first set of results presents a comparison between the available SNR in MFNs and SFNs using HPHT topologies. Three different frequency reuse patterns are selected for the MFN $(N=1,3,4)$ whereas for the SFN an ideal large area $\mathrm{SFN}$ is designed. For the latter case different $\mathrm{CP}$ duration $\left(T_{g}\right.$ $=33 \mu \mathrm{s}, 100 \mu \mathrm{s}, 200 \mu \mathrm{s}$ and $400 \mu \mathrm{s})$ are computed. The results are computed for the $10^{t h}, 5^{t h}$ and $1^{s t}$ percentiles. Table IV collects the SINR values achieved in the MFN and SFN cases.

According to the results, decreasing frequency reuse $N$ involves, as expected, a decrease in the available SINR, which is particularly dramatic for $N=1$ since in this case all sites except the wanted are interfering. The differences between the $10^{\text {th }}$ to $5^{\text {th }}$ and $5^{\text {th }}$ to $1^{\text {st }}$ percentiles show a higher slope for the $N=1$ (3.4 dB and $6 \mathrm{~dB}$ differences, respectively) than for $N=3$ and 4 ( $2.9 \mathrm{~dB}$ and $5.2 \mathrm{~dB}$ differences, in both cases). The SFN results outperform MFN $N=1$ results even for the shortest $33 \mu \mathrm{s}$ CP duration in which the amount of SFN selfinterference is lower than the interference in the MFN case. Increasing CP duration to $T_{g}=100 \mu \mathrm{s}$ and $T_{g}=200 \mu$ s provide SINR values similar as those achieved in the MFN $N=3$ and 4 , respectively.

Figure 6 depicts the CDFs of different network configurations. It can be seen that the distribution for the SFN with $T_{g}$ $=200 \mu \mathrm{s}$ and the MFN with $N=4$ are similar except for the lower tails, in which the SFN outperforms the MFN thanks to the so-called SFN gain. The SFN gain is translated into a decrease in the standard deviation of the SINR distribution in the CoI. According to the values in Table IV, it can be seen that the slope of the $\mathrm{CDF}$ is more abrupt for low $\mathrm{CP}$ duration than for increasing duration. The highest slope is found between $T_{g}$ $=33 \mu \mathrm{s}$ and $T_{g}=100 \mu \mathrm{s}$ what suggests that a large number of stations are included as useful contributions when increasing the $\mathrm{CP}$ duration, being the network in the first case highly selfinterference limited. In general, a given coverage percentage is reached faster with an SFN than with an MFN.

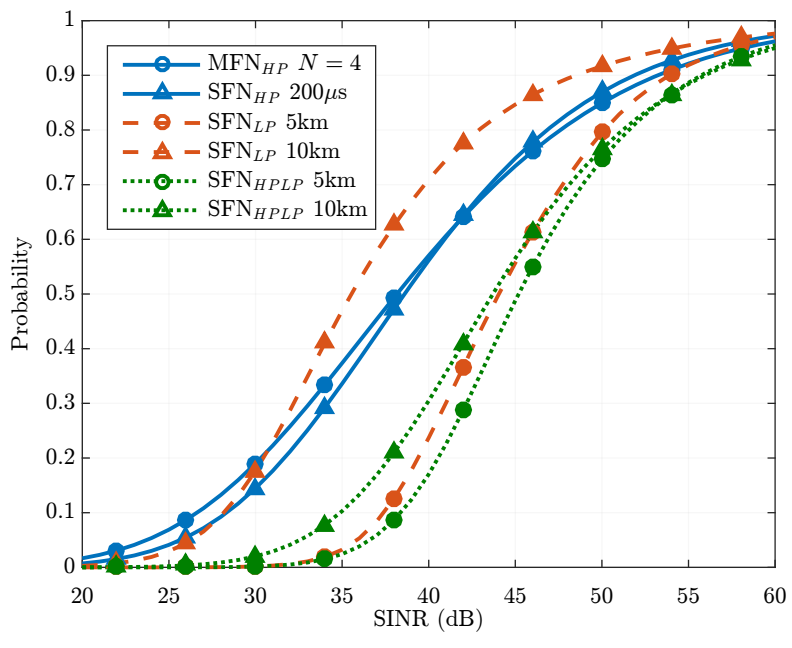

Fig. 6. Comparison between the CDFs of different networks configurations: MFN HPHT and SFN HPHT, LPLT and mixed HPHT+LPLT. The SFN curves are configured with CP duration $T_{g}=200 \mu \mathrm{s}$. SFNs involving LPLT sites are computed for ISD $5 \mathrm{~km}$ and $10 \mathrm{~km}$.

\section{B. Comparison between LPLT SFNs}

The behavior of the SINR distributions are studied for SFNs built up with LPLT sites.

Figure 7 shows the available SINR values in an SFN LPLT deployment (solid lines) for different ISD values $(I S D=3,5$, 10 and $20 \mathrm{~km}$ ). A CP duration of $200 \mu \mathrm{s}$ is selected so that the network is not strongly self-interference limited. The results show that SFN LPLT deployments with low ISD outperform the coverage of a HPHT SFN with the largest CP duration.

Regarding the slope of the CDF shown in Figure 6 (and the differences between the $10^{\text {th }}, 5^{\text {th }}$ and $1^{\text {st }}$ percentiles) it can be seen that it is more abrupt for short ISD, decreasing for larger ISDs. Thus, a given coverage percentage is faster reached with short ISD. The LPLT SFN with $I S D_{L P}=10 \mathrm{~km}$ provides the closest result to the HPHT MFN network with $N=4$. For sparse networks (e.g. $I S D=20 \mathrm{~km}$ ), the SINR values decrease, even below the SINR values achieved by the HPHT SFN with $T_{g}=100 \mu \mathrm{s}$.

\section{Mixed LPLT+HPHT networks}

One step further consist of considering a mixed HPHT and LPLT network in which both network infrastructures can be used by covering one HPHT MFN cell with an SFN deployment of LPLT sites.

Figure 7 also depicts the results for a combined HPHT and LPLT network using a CP duration of $200 \mu \mathrm{s}$. As in the only LPLT case, the highest SINR values are achieved by short ISDs $(5 \mathrm{~km}$ or $10 \mathrm{~km}$ ) even outperforming the LPLT result thanks to the contribution of the HPHT network. However, in these cases, the gain is not substantial.

On the other hand, for sparse LPLT deployments (e.g. $10 \mathrm{~km}$ and $20 \mathrm{~km}$ ) the improvement is significant since the underlying HPHT network already covers a high number of locations which are not covered by a sparse deployment. The effort in SINR for increasing the coverage from the $10^{\text {th }}$ to the $1^{\text {st }}$ 


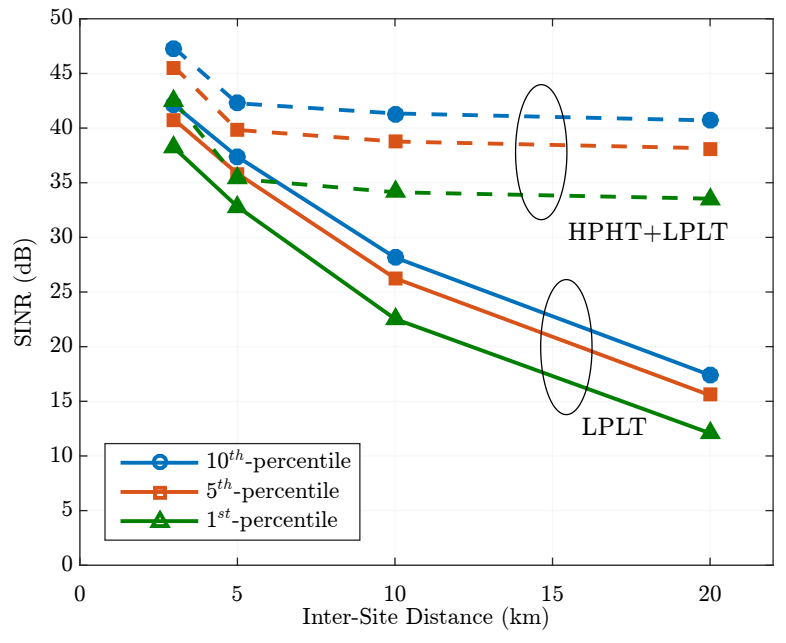

Fig. 7. Comparison between available SINR in LPLT (solid line) and mixed HPHT+LPLT (dashed line) SFNs for CP duration $T_{g}=200 \mu \mathrm{s}$. SINR values at $10^{t h}, 5^{t h}$ and $1^{\text {st }}$ coverage percentiles are shown.

percentile is higher than with only LPLT, see Figure 6, due to the already large SINR values achieved by the HPHT site. Moreover, Figure 7 also shows that the differences in available SINR for ISD larger than $5 \mathrm{~km}$ are in the order of 1 or $2 \mathrm{~dB}$. This suggests that when using a HPHT station in combination with a LPLT network not all stations need necessarily to be active to achieve high SINR values.

\section{Case Study: CP duration evaluation under a realistic scenario}

A hexagonal lattice has been used to validate the described methodology. A more realistic scenario is used in this exercise for comparison with the calculations provided by a professional planning tool ${ }^{1}$. The practical scenario consist of a real DTT station (Olympiaturm) located in Munich, Germany. A series of LPLT sites have been deployed around the service area of the DTT station, covering populated areas far from this station. The scenario under evaluation results in an heterogeneous deployment with non-uniform ISD and site height. The SINR offered by the HPHT station will be evaluated in isolation and for outdoor portable reception conditions. The network defined by the LPLT sites is connected to the main station, by means of SFN, to calculate the potential improvement in available SINR and, thus, outdoor portable coverage. The network layout is depicted in Figures 8 and 9 , see the black triangles representing sites being the one at the center the HPHT station. Relevant network parameters and reception conditions are those listed in Table I except the following changes: frequency (594 MHz), ERP HPHT $(40 \mathrm{~kW})$, ERP LPLT $(1 \mathrm{~kW})$, HPHT antenna height $(258$ $\mathrm{m})$. Portable outdoor reception is modeled with an omnidirectional receiving antenna with $0 \mathrm{~dB}_{i}$ gain, $16.38 \mathrm{~dB}$ height loss. Note that feeder loss is set to $0 \mathrm{~dB}$.

\footnotetext{
${ }^{1}$ The planning tool used for this exercise is FRANSY, a frequency-analysis software tool for planning terrestrial broadcast networks developed by the Institut für Rundfunktechnik (IRT), Germany.
}

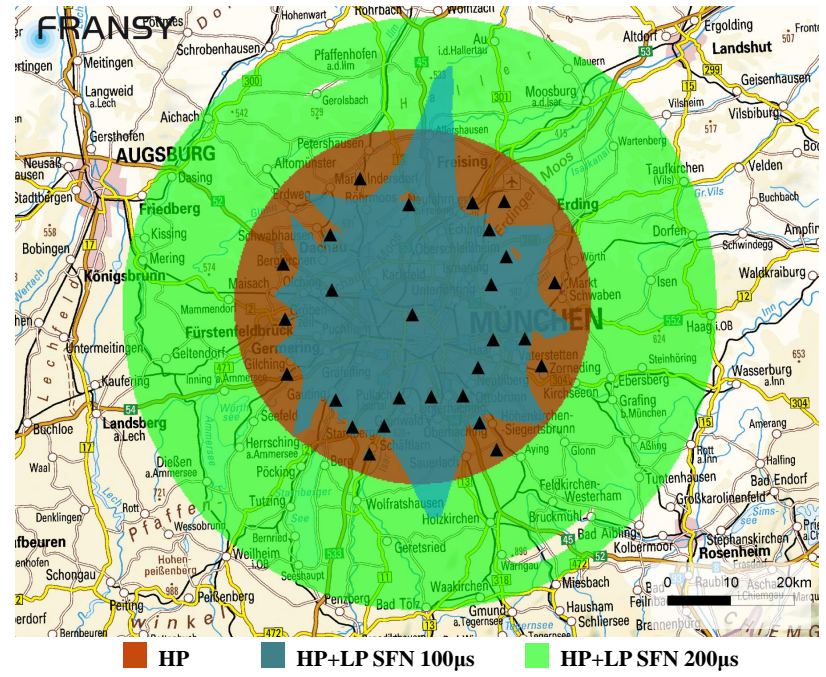

Fig. 8. Comparison between the areas covered with SINR greater than $20 \mathrm{~dB}$ for the isolated HPHT, and HPHT+LPLT SFNs with $100 \mu \mathrm{s}$ and $200 \mu \mathrm{s}$ CP duration. Portable outdoor reception conditions. Underlying map: (C) GeoBasis-DE/BKG 2010

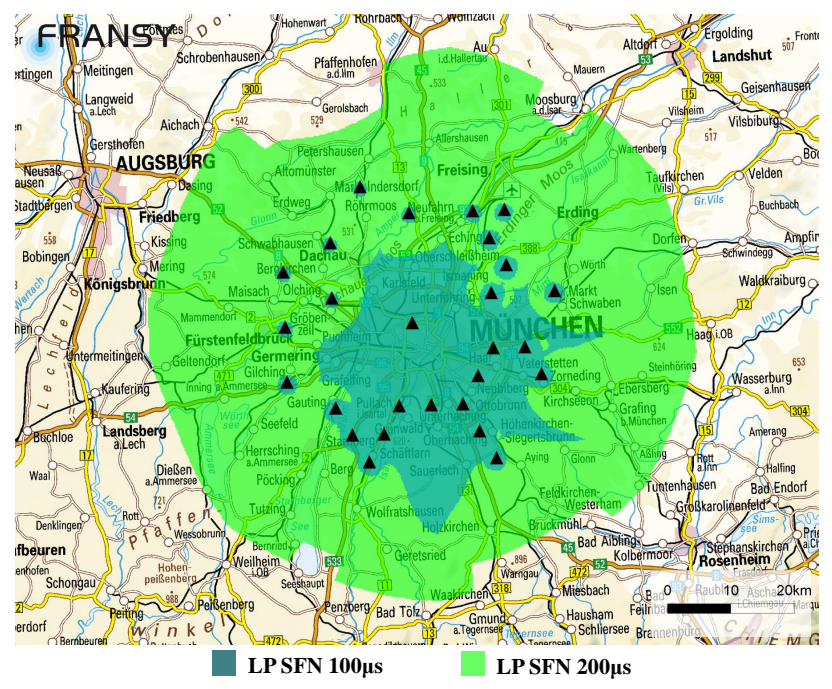

Fig. 9. Comparison between the areas covered with SINR greater than $20 \mathrm{~dB}$ for the LPLT SFNs with $100 \mu \mathrm{s}$ and $200 \mu \mathrm{s}$ CP duration. Portable outdoor reception conditions. Underlying map: (C) GeoBasis-DE/BKG 2010

Figures 8 and 9 show the footprints using the planning tool for different network configuration involving HPHT, LPLT with 100 and $200 \mu \mathrm{s} \mathrm{CP}$ duration, and joint HPHT+LPLT deployments for an available SINR equal to or greater than $20 \mathrm{~dB}$.

It can be seen that the covered area by the HPHT station is limited due to the severe path loss for portable outdoor reception in comparison with the deployments that include LPLT sites by means of SFN. The use of SFN on the LPLT sites with $100 \mu \mathrm{s}$ is appropriate to cover sparse ISDs although with an important presence of SFN self-interference. The use of $200 \mu$ s already exceeds the area covered by the HPHT station. The connection of the HPHT via SFN provides increased coverage area which can even compensate for the lack of enough CP. 


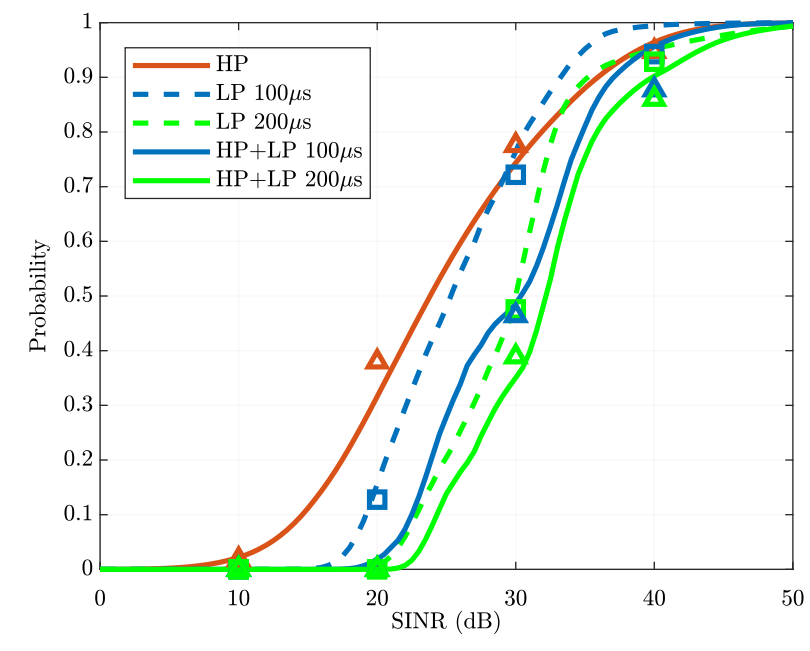

Fig. 10. Comparison of results provided by the semianalytical CDF and a series of points extracted from the professional network planning tool at 10 , 20, 30 and $40 \mathrm{~dB}$, represented by markers.

A more extensive analysis is provided in Figure 10 where the results from the semianalytical method and the values offered by the planning tool are compared. The comparison is performed in terms of percentage of covered area over a service area defined by a radius of $50 \mathrm{~km}$. Planning tools generally provide results in terms of footprints, thus, it is not possible to directly obtain the CDF. Instead, values at 10, 20, 30 and $40 \mathrm{~dB}$ are obtained, which are represented by markers.

The prediction is particularly accurate for the tail of the CDF whereas discrepancies of several $\mathrm{dBs}$ can be found at the upper part of the curves, which are less critical for the selection of a proper transmission mode to maximize coverage. As expected, the connection of HPHT+LPLT sites provide the best performance although the use of LPLT with the appropriate CP already outperforms HPHT coverage.

\section{Conclusions}

This paper has presented a semianalytical method for deriving the SINR distribution in broadcast SFN networks combining both HPHT and LPLT sites. Instead of Monte Carlo simulations, the proposed solution may reduce the computation complexity especially when a large number of stations are considered over a large area SFN.

The method is generic so that it can be applied to real deployments combining HPHT+LPLT stations. Moreover, its application to hexagon lattice deployments permits to obtain a coarse estimate of the most suitable $\mathrm{CP}$ duration for the broadcast system to be implemented and the influence of other network parameters, such as ISD or transmit power, in the available coverage and capacity.

The accuracy of the results can be improved by the selection of other propagation methods and log-normal summation approaches. The results show that the k-LNM method is valid for both HPHT and LPLT networks, although a penalty in accuracy is obtained in LPLT SFNs with respect to HPHT SFNs and MFNs in the low tail of the curves. This could be further improved by considering the potential correlation between useful and interfering contributions in the SFN.

The main conclusions derived from the analysis with different network infrastructure are the following:

- Dense LPLT SFNs (e.g. urban deployments) outperform the SINR of a HPHT MFN network whereas sparse LPLT SFNs (e.g. rural deployments) cannot provide the same coverage.

- The combination of HPHT and LPLT infrastructures is possible as soon as a large CP duration (at least $100 \mu \mathrm{s}$ ) is guaranteed.

- Dense enough deployments may already outperform the coverage provided by HPHT networks. On the other hand, for sparse LPLT networks, the introduction of a HPHT is beneficial since it can increase the SINR beyond that provided by an HPHT MFN as well as a LPLT-only SFN.

\section{ACKNOWLEDGEMENTS}

The authors would like to thank the FRANSY development team at the Institut für Rundfunktechnik (IRT) for their valuable contribution to the studies presented in this paper.

\section{REFERENCES}

[1] A. Ligeti, "Single Frequency Network Planning," Ph.D. dissertation, Kungliga Tekniska Högskolan, 1999.

[2] I. Eizmendi, M. Velez, D. Gómez-Barquero, J. Morgade, V. BaenaLecuyer, M. Slimani, and J. Zoellner, "DVB-T2: The Second Generation of Terrestrial Digital Video Broadcasting System," IEEE Transactions on Broadcasting, vol. 60, no. 2, pp. 258-271, June 2014.

[3] L. Fay, L. Michael, D. Gómez-Barquero, N. Ammar, and M. W. Caldwell, "An Overview of the ATSC 3.0 Physical Layer Specification," IEEE Transactions on Broadcasting, vol. 62, no. 1, pp. 159-171, March 2016.

[4] "Delivery of Broadcast Content over LTE Networks," European Broadcasting Union, Tech. Rep. 027, Jul 2014.

[5] C. Hoymann, D. Astely, M. Stattin, G. Wikstrom, J. F. Cheng, A. Hoglund, M. Frenne, R. Blasco, J. Huschke, and F. Gunnarsson, "LTE release 14 outlook," IEEE Communications Magazine, vol. 54 no. 6, pp. 44-49, June 2016.

[6] L. Zhang, Y. Wu, G. K. Walker, W. Li, K. Salehian, and A. Florea, "Improving LTE eMBMS With Extended OFDM Parameters and LayeredDivision-Multiplexing," IEEE Transactions on Broadcasting, vol. 63, no. 1, pp. 32-47, March 2017.

[7] U. Meabe, X. Gil, C. Li, M. Vélez, and P. Angueira, "On the Coverage and Cost of HPHT Versus LPLT Networks for Rooftop, Portable, and Mobile Broadcast Services Delivery," IEEE Transactions on Broadcasting, vol. 61, no. 2, pp. 133-141, June 2015.

[8] L. Shi, K. W. Sung, and J. Zander, "Future TV Content Delivery Over Cellular Networks From Urban to Rural Environments," IEEE Transactions on Wireless Communications, vol. 14, no. 11, pp. 61776187, Nov 2015.

[9] S. Mukherjee, "Distribution of Downlink SINR in Heterogeneous Cellular Networks," IEEE Journal on Selected Areas in Communications, vol. 30, no. 3, pp. 575-585, April 2012.

[10] H. S. Jo, Y. J. Sang, P. Xia, and J. G. Andrews, "Heterogeneous Cellular Networks with Flexible Cell Association: A Comprehensive Downlink SINR Analysis," IEEE Transactions on Wireless Communications, vol. 11, no. 10, pp. 3484-3495, October 2012.

[11] H. S. Dhillon, R. K. Ganti, F. Baccelli, and J. G. Andrews, "Modeling and Analysis of K-Tier Downlink Heterogeneous Cellular Networks," IEEE Journal on Selected Areas in Communications, vol. 30, no. 3, pp. 550-560, April 2012.

[12] K. Sung, H. Haas, and S. McLaughlin, "A Semianalytical PDF of Downlink SINR for Femtocell Networks," EURASIP Journal on Wireless Communications and Networking, vol. 2010, no. 1, pp. 256-370, 2010.

[13] J. A. Arenas, U. Gil, D. Plets, P. Angueira, W. Joseph, and L. Martens, "Statistical Analysis of Field Strength Location Variability for UHF Multimedia Broadband Services," IEEE Antennas and Wireless Propagation Letters, vol. 11, pp. 34-36, 2012. 
[14] "Frequency and Network Planning Aspects of DVB-T2," European Broadcasting Union, Tech. Rep. 3384, Nov 2013.

[15] K. Beeke, "Spectrum Planning: Analysis of Methods for the Summation of Log-Normal Distributions," EBU Technical Review, pp. 1-9, Oct 2007.

[16] L. Fenton, "The Sum of Log-Normal Probability Distributions in Scatter Transmission Systems," IRE Transactions on Communications Systems, vol. 8, no. 1, pp. 57-67, March 1960.

[17] S. C. Schwartz and Y. S. Yeh, "On the Distribution Function and Moments of Power Sums With Log-Normal Components," Bell System Technical Journal, vol. 61, no. 7, pp. 1441-1462, 1982.

[18] A. Safak, "Statistical analysis of the power sum of multiple correlated log-normal components," IEEE Transactions on Vehicular Technology, vol. 42, no. 1, pp. 58-61, Feb 1993.

[19] N. C. Beaulieu and Q. Xie, "An optimal lognormal approximation to lognormal sum distributions," IEEE Transactions on Vehicular Technology, vol. 53, no. 2, pp. 479-489, March 2004.

[20] S. B. Slimane, "Bounds on the distribution of a sum of independent lognormal random variables," IEEE Transactions on Communications, vol. 49, no. 6, pp. 975-978, Jun 2001.

[21] "Simulation Parameters for Theoretical LTE eMBMS Network Studies," EBU, Tech. Rep. 034, Dec 2015.

[22] "Method for point-to-area predictions for terrestrial services in the frequency range $30 \mathrm{MHz}$ to $3000 \mathrm{MHz}$," ITU, Recommendation ITU-R P.1546-5, 2013

[23] "Directivity and polarization discrimination of antennas in the reception of television broadcasting," ITU, Recommendation ITU-R BT.419-3.

[24] R. Brugger and D. Hemingway, "OFDM Receivers. Impact on Coverage of Inter-Symbol Interference and FFT Window Positioning," EBU, Tech. Rep. Jul, 2003.

[25] T. M. Cover and J. A. Thomas, Elements of Information Theory. John Wiley \& Sons, Inc., 2005.

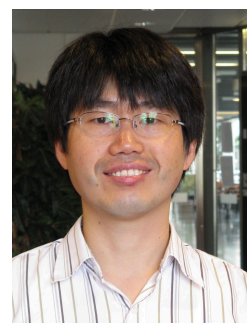

Ki Won Sung (M'10) is a Docent researcher in the Communication Systems Department at KTH Roya Institute of Technology, Stockholm, Sweden. He is also affiliated with KTH Center for Wireless Systems (Wireless@kth). He received a B.S. degree in industrial management, and M.S. and Ph.D. degrees in industrial engineering from Korea Advanced Institute of Science and Technology (KAIST) in 1998, 2000, and 2005, respectively. From 2005 to 2007 he was a senior engineer in Samsung Electronics, Korea, where he participated in the development and commercialization of a mobile WiMAX system. In 2008 he was a visiting researcher at the Institute for Digital Communications, University of Edinburgh, United Kingdom. He joined KTH in 2009. He has participated in several European collaboration projects such as QUASAR, METIS, and METIS-II. His research interests include future media delivery platform, 5G wireless architecture, energy-efficient wireless networks, and technoeconomics of wireless systems.

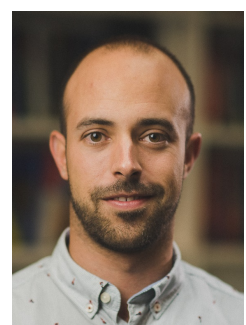

Jordi Joan Gimenez received a M.Sc. degree in Telecommunications engineering from the Universitat Politcnica de Valncia (UPV) in 2010, and a $\mathrm{Ph} . \mathrm{D}$. degree in Telecommunication from the UPV in 2015 .

$\mathrm{He}$ is a Post-Doc Researcher (Generalitat Valenciana Fellow) at iTEAM-UPV, within the research group working on terrestrial broadcasting and the optimization of next-generation broadcast systems. During his doctoral studies, he was a Guest researcher at the Royal Institute of Technology (Stockholm, Sweden), and at Teracom AB (Stockholm, Sweden), the Swedish Digital Terrestrial TV operator. He has been a Post-Doctoral Guest Researcher at the Institut für Rundfunktechnik (Munich, Germany) in 2015.

Dr. Gimenez has participated in the standardization process of the nextgeneration handheld standard DVB-NGH, as well as in the DVB technical group working on the assessment of transmission technologies for the next generation terrestrial broadcasting (DVB TM-T MIMO Study Mission). Part of the results of his research has also been presented in the ATSC 3.0 standardization process, within the Waveform Ad-Hoc group.

His main research interests include the network and frequency planning of next-generation terrestrial broadcast networks for an optimal spectrum usage. His research on the WiB (Wideband Reuse-1) concept, together with Teracom and Panasonic, was awarded with the 2016 IBC Best Conference Paper Award.

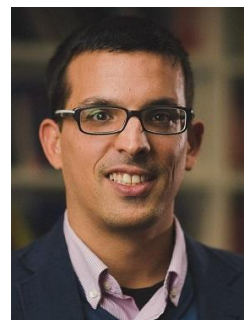

David Gomez-Barquero received the double M.Sc. degrees in telecommunications engineering from the Universitat Politecnica de Valencia (UPV), Spain, and the University of Gävle, Sweden, in 2004, the $\mathrm{Ph} . \mathrm{D}$. degree in telecommunications from the UPV in 2009; and he carried out a 2-year post-doc at the Fraunhofer Heinrich Hertz Institute, Germany.

$\mathrm{He}$ is a Senior Researcher (Ramon \& Cajal Fellow) with the Institute of Telecommunications and Multimedia Applications, UPV, where he leads a research group working on next generation broadcasting technologies. Previously, he hold visiting research appointments at Ericsson Eurolab, Germany, the Royal Institute of Technology, Sweden, the University of Turku, Finland, the Technical University of Braunschweig, Germany, the Sergio Arboleda University of Bogota, Colombia, and the New Jersey Institute of Technology, USA.

Dr. Gomez-Barquero has been since 2008 actively participating in the digital television standardization, including DVB-T2, T2-Lite, DVB-NGH, and ATSC 3.0, acting as Vice-Chairman of the Modulation and Coding Ad-Hoc Group. He is the Editor of the book entitled Next Generation Mobile Broadcasting (CRC Press, 2013), and Associate Editor of the IEEE TRANSACTIONS ON BROADCASTING. 